\title{
Novel bi-allelic mutations in DNAH1 cause multiple morphological abnormalities of the sperm flagella resulting in male infertility
}

\author{
Chuan Jiang ${ }^{1}$, Xueguang Zhang ${ }^{1}$, Heng Zhang ${ }^{2}$, Junliang Guo ${ }^{3,4}$, Chaoliang Zhang ${ }^{5}$, Jinhong Li ${ }^{3,4} \wedge$, \\ Yihong Yang ${ }^{3,4}$
}

${ }^{1}$ Department of Obstetrics/Gynecology, Joint Laboratory of Reproductive Medicine (SCU-CUHK), Key Laboratory of Obstetric \& Gynecologic and Pediatric Diseases and Birth Defects, Ministry of Education, Sichuan University, Chengdu, China; ${ }^{2}$ Department of Otolaryngology, The First People's Hospital of Longquanyi District of Chengdu, Chengdu, China; ${ }^{3}$ Center of Reproductive Medicine, West China Second University Hospital, Sichuan University, Chengdu, China; ${ }^{4}$ Key Laboratory of Obstetric \& Gynecologic and Pediatric Diseases and Birth Defects, Ministry of Education, Sichuan University, Chengdu, China; ${ }^{5}$ State Key Laboratory of Oral Diseases, West China Hospital of Stomatology, Sichuan University, Chengdu, China

Contributions: (I) Conception and design: J Li, Y Yang, C Jiang, X Zhang; (II) Administrative support: None; (III) Provision of study materials or patients: J Li, Y Yang; (IV) Collection and assembly of data: C Jiang, X Zhang, J Guo, C Zhang; (V) Data analysis and interpretation: C Jiang, X Zhang, H Zhang; (VI) Manuscript writing: All authors; (VII) Final approval of manuscript: All authors.

Correspondence to: Jinhong Li; Yihong Yang. Center of Reproductive Medicine, West China Second University Hospital, Sichuan University, Chengdu, China; Key Laboratory of Obstetric \& Gynecologic and Pediatric Diseases and Birth Defects, Ministry of Education, Sichuan University, Chengdu 610041, China. Email: cd1202012@163.com; andrologyhong1966@163.com.

Background: Male infertility is a major health concern and approximately $10-15 \%$ of cases are caused by genetic abnormalities. Defects in the sperm flagella are closely related to male infertility, since flagellar beating allows sperm to swim. The sperm of males afflicted with multiple morphological abnormalities of the flagella (MMAF) possess severe defects of the sperm flagella, may impair sperm motility and lead to male infertility. Currently, known genetic defects only account for MMAF in about $60 \%$ of patients and need more intensive efforts to explore the relationship between genes and MMAF.

Methods: The whole-exome sequencing (WES) was performed to analyze the genetic cause of the MMAF patient. Scanning electron microscopy (SEM) and transmission electron microscopy (TEM) were used to observe the morphology of sperm cells and to identify the ultrastructural characteristics of the flagella in the patient. The expression of DNAH1 was analyzed by sperm immunofluorescence staining.

Results: We identified the negative effects produced by the DNAH1 mutations c. $8170 . \mathrm{C}>\mathrm{T}$ (p. R2724*) and c. 4670C>T (p. T1557M) on DNAH1 expression and the development of sperm flagella.

Conclusions: Our findings suggest that DNAH1 is associated with the formation of sperm flagella and homozygous loss-of-function mutations in DNAH1 can impair sperm motility and cause male infertility.

Keywords: Multiple morphological abnormalities of the flagella (MMAF); whole-exome sequencing (WES); male infertility

Submitted Nov 17, 2020. Accepted for publication Feb 18, 2021.

doi: $10.21037 /$ tau-20-1434

View this article at: http://dx.doi.org/10.21037/tau-20-1434

^ ORCID: 0000-0002-7814-136X. 


\section{Introduction}

Male infertility is a major health concern and approximately $10-15 \%$ of cases are caused by genetic abnormalities (1). Defects in the sperm flagella are closely related to male infertility, since flagellar beating allows sperm to swim. The sperm of males afflicted with multiple morphological abnormalities of the flagella (MMAF) presents severe defects, including bent, short, coiled, absent, and/or irregular flagella that may fully or partially impair sperm motility. The cytoskeleton of motile flagella possesses a $(9+2)$ axonemal configuration, consisting of nine outer microtubule doublets (ODs) and two central microtubules (CPs) surrounded by fibrous sheath (FS) and outer dense fiber (ODF) (2). The typical features of MMAF include an abnormal ultrastructure of axons and absence of ODF and the FS in the flagella, as well as stump or short tails and dysplasia of the fibrous sheath (DFS), causing partial or complete asthenozoospermia.

Developmental disturbances may be responsible for abnormalities in mitochondrial sheath, failure of the flagella to properly elongate, and the characteristic absence of $\mathrm{CPs}$ in MMAF with patients. The development of MMAF has been linked to many genes, including CFAP65, CFAP44, CFAP43, CFAP69, DNAH1, CFAP251, AK7, CEP135, QRICH2, FSIP2, TTC21A, SPEF2, ARMC2, WDR66, $A K A P 4$, and CCDC39 (3-19). However, known genetic defects account for only $60 \%$ of patients with MMAF. The causes of the remaining MMAF cases remain unknown, which could indicate uncharacterized genetic defects. Therefore, the relationship between genetic abnormalities and MMAF should be further scrutinized.

DNAH1 (MIM 603332), which encodes an inner dynein arm heavy chain, is the first axonemal gene responsible for infertility phenotype without any of the other symptoms usually observed in primary ciliary dyskinesia (PCD) and is also highly associated with immotile sperm. The DNAH1 protein connects the outer doublet and radial spokes of sperm, while the central doublets are localized and stabilized by the radial spokes (20). Pathogenic mutations or the absence of DNAH1 can result in the absence or dysfunction of the central doublets in the axoneme. Lossof-function mutations in DNAH1 are known to lead to MMAF in humans and mice, seeing that they are harmful to the organization and biogenesis of the sperm axoneme (6).

There is limited information on the pathogenic variants of DNAH1 in the Chinese population, and understanding this association may lead to a greater understanding of
MMAF. We discovered a hereditary homozygous DNAH1 mutation in a patient with MMAF and successfully identified a novel homozygous nonsense mutation in $D N A H 1$ by whole-exome sequencing (WES). We identified the negative effects produced by the DNAH1 mutations c. $8170 . \mathrm{C}>\mathrm{T}$ (p. R2724*) and c. $4670 \mathrm{C}>\mathrm{T}$ (p. T1557M) on DNAH1 expression and the development of sperm flagella. Our findings suggest that DNAH1 is related to the formation of sperm flagella, and that homozygous mutations in DNAH1 can damage sperm motility and lead to male infertility. We present the following article in accordance with the MDAR reporting checklist (available at http://dx.doi.org/10.21037/tau-20-1434).

\section{Methods}

\section{Study participants}

A 28-year-old Han Chinese man was diagnosed with infertility 2 years before our study, who was from a consanguineous family. The patient's wife did not have any fertility-related disorders, and his parents were also evaluated. The control sample included 200 unrelated Han Chinese male volunteers, who had naturally conceived at least one child and had normal sperm quality according to the World Health Organization index (WHO-5) (sperm concentration $\geq 15$ million $/ \mathrm{mL}$; progressively motile sperm $\geq 32 \%$; normal sperm morphology $\geq 4 \%$ ). The study was conducted in accordance with the Declaration of Helsinki (as revised in 2013). The study was approved by the ethics committee of West China Second University Hospital (No. 2019019) and informed consent was taken from the patient and all members of his family.

\section{WES and Sanger sequencing}

The DNA of the patient was analyzed using WES. Briefly, genomic DNA was separated from peripheral blood samples using the FitAmp Plasma/Serum DNA Isolation Kit (EpiGentek), exon capture was performed using the the Agilent SureSelect Human All Exon V6 Kit, and an Illumina HiSeq X system was used for sequencing. ANNOVAR was used for functional annotation and further filtering was performed using the 1000 Genomes Project, Human Gene Mutation Database (HGMD), database of single nucleotide polymorphisms (dbSNP), and Exome Aggregation Consortium (ExAC). After filtering, functional predictions for the retained nonsynonymous single nucleotide variants 
(SNVs) were performed using PolyPhen-2 (http://genetics. bwh.harvard.edu/pph2), SIFT (https://sift.bii.a-star.edu. sg), MutationTaster, and CADD. Using Sanger sequencing, the patient's parents, as well as the control subjects, were tested for the candidate pathogenic gene variants identified in the patient. The ProFlex PCR System (Thermo Fisher Scientific) was used for polymerase chain reaction (PCR) amplification and the DNA sequencing of PCR products was conducted on an ABI377A DNA sequencer (Applied Biosystems). The PCR primers were: DNAH1-1: 5'-GGC CAACCTCATGGCTGCTTACACAG-3' and 5'-CCAAG GGCCTGAGCTTCAGCAAGAC-3', DNAH1-2: 5'-CC GTGAGGAGGCCTCAGAGGAAACTC-3' and 5'-CTG TCCTTGAGGTTCTGGGACAAGC-3'.

\section{Immunofluorescence staining}

The sperm samples were fixed with $4 \%$ paraformaldehyde, permeabilized with $0.3 \%$ Triton $\mathrm{X}-100$, and sealed with $5 \%$ bovine serum albumin (BSA); they were then incubated with primary antibodies (1:50, DNAH1, sc-102481, Santa Cruz; 1:50, CL48866031, $\alpha$-tubulin, Proteintech) overnight at $4{ }^{\circ} \mathrm{C}$. On the next day, samples were washed with $1 \times$ PBS three times, incubated with DyLight 488-labeled secondary antibodies (1:800, 1927937, Thermo Fisher Scientific) for $1 \mathrm{~h}$ at $25^{\circ} \mathrm{C}$, and then the nuclei were stained with 4,6-diamidino-2-phenylindole (DAPI; Sigma-Aldrich). Images were obtained using laser scanning confocal microscopy (Olympus).

\section{Electron microscopy}

The sperm samples were prepared using Sperm Washing Medium (SpermRinse, Vitrolife), mounted onto slides, and evaluated using scanning electron microscopy (SEM) and transmission electron microscopy (TEM).

\section{Results}

\section{MMAF phenotype identified in an infertile patient}

The proband, a 28-year-old man from a consanguineous family, had been diagnosed with infertility 2 years before our study. His semen samples were submitted to examination three times. As shown in Table 1, the sperm count was a very low, and most cells were immobile because of the completely defective flagella. These abnormalities were confirmed by Papanicolaou staining. Specifically, spermatozoa from the patient displayed typical MMAF phenotypes, including short, absent, coiled flagella with an irregular caliber, rather than the long, thin tails of normal sperm (Figure $1 A$ ). A more vivid MMAF phenotype was revealed via SEM (Figure 1B), and the patient's spermatozoa were further analyzed using TEM. Remarkably, the normal sperm flagella consists of $9+2$ axons, including nine ODs and two CPs, ODF and FS; this patient's microtubules showed a significant disorder in the axons and other surrounding components. The abnormalities included missing CPs, and disordered OD arrangements in the midpiece, principal, and end pieces (Figure 1C). Therefore, we speculate that the observed MMAF phenotype is the cause of infertility in this patient.

\section{Bi-allelic loss-of-function mutations of DNAH1 in an infertile male with MMAF}

To elucidate the genetic cause of MMAF, our study performed WES analysis on this affected male subject. Based on the autosomal-recessive inheritance of MMAF, variants were excluded if the following conditions were met: (I) in the ExAC Browser, gnomAD or the 1000 Genome Project, minor allele frequency greater than or equal to $1 \%$, because the pathogenic variants that cause MMAF are rare in humans; (II) no harmful variant detected using SIFT, PolyPhen-2, or Mutation Taster tools; (III) except for the canonical splice sites, all variants located in noncoding exons, 30 or 50 untranslated regions, or intronic sequences; (IV) the variant is heterozygous. As a result, we identified two DNAH1 bi-allelic pathogenic variants in the MMAFaffected man: [c. 8170.C>T (p. R2724*) and c. 4670C>T (p. $\mathrm{T} 1557 \mathrm{M})$ ] (Figure 2A), and these mutations were further verified through Sanger sequencing of this patient and his parents (Figure $2 B$ ). Notably, these bi-allelic pathogenic variants in DNAH1 were not detected in the 200 normal controls, and the two mutation sites are $100 \%$ conserved among many species (Figure 2C). Therefore, we conclude that these bi-allelic variants in DNAH1 were the cause of MMAF in this patient.

\section{Effects of the bi-allelic variants on the DNAH1 protein}

To determine the negative effects of the two variants on DNAH1 expression, we performed immunofluorescence analysis on the sperm samples of patients and normal controls using an anti-DNAH1 antibody. As expected, fulllength DNAH1 was detected in the sperm flagella of the 
Table 1 Semen parameters of patient and normal control

\begin{tabular}{lccc}
\hline Semen parameters & Patient & Normal control & Normospermic parameters \\
\hline Sperm volume $(\mathrm{mL})$ & 4.8 & 3.5 & $\geq 1.5$ \\
Sperm concentration (million/mL) & 1.8 & 61 & $\geq 15$ \\
Motility sperm (\%) & 1 & 52 & $\geq 40$ \\
Vitality (\%) & 1 & 75 & $\geq 58$ \\
Normal spermatozoa (\%) & 0.5 & 2.3 & - \\
Absent flagella (\%) & 31 & 9 & - \\
Short flagella (\%) & 25 & 8.5 & - \\
Coiled flagella (\%) & 13.5 & 3.7 & - \\
Bent flagella (\%) & 3 & 1.5 & - \\
Flagella of irregular caliber (\%) & 27 & & - \\
\hline
\end{tabular}

control group (Figure 3A). However, DNAH1 staining was hardly detected in the spermatozoa of the patient, indicating that the absence of DNAH1 expression was probably caused by the bi-allelic variants. To uncover the mechanism that led to a lack of DNAH1 expression, we analyzed the characteristics of the bi-allelic variants. The c. 8170 . C>T (p. R2724*) mutation, which results in a truncated protein owing to a premature termination codon, can be clearly linked to protein degradation. On the other hand, c. $4670 \mathrm{C}>\mathrm{T}$ (p. $\mathrm{T} 1557 \mathrm{M}$ ) is a missense mutation that, may lead to DNAH1 protein degradation by mechanisms other than affecting the transcription or translation processes. Considering that DNAH1 is a vital structural protein, we hypothesized that this mutation may change protein conformation. Therefore, we predicted the conformational changes in the DNAH1 protein induced by this mutation using SWISSMODEL4.0 (https://swissmodel.expasy. org/) and PyMoL software 4.1 (1.3r1, DeLano Scientific LLC). Strikingly, the molecular modeling showed that the spatial structure of $D N A H 1$ was completely changed by this mutation, including random coils, $\alpha$-helices and $\beta$-sheets (Figure 3B). Altogether, our results showed that the deficiency in DNAH1 expression was induced by the bi-allelic loss of function mutations that led to an MMAF phenotype in this infertile man.

\section{Discussion}

In 2001, asthenozoospermia owing to DNAH1 mutations were identified (4). MMAF became a recognized disorder in 2014, and several pathogenic variants of DNAH1 associated with sperm flagella development were recognized as potential contributors to MMAF (5,7,21-24). We identified bi-allelic loss-of-function mutations in DNAH1 from an infertile male with MMAF by WES. This mutation led to the absence of DNAH1 protein in sperm flagella resulting in abnormal flagellar development and irregular sperm ultrastructure.

The DNAH1 gene is composed of 79 exons, located on chromosome 3 p21.1, with a size of $13,126 \mathrm{bp}$. In spermatozoa, DNAH1 is involved in the formation of the internal dynamic armis, which consists of seven molecular complexes arranged in a 3-2-2 fashion into three different types of internal dynamic arms (IDA1 to IDA3). DNAH1 is an integral part of IDA3, which provides an anchoring site for radial spoke 3 (RS3). Missing outer dynein arms suggest that DNAH1 may localize between RS3 and outer dynein arms on microtubules (25). The absence of DNAH1 results in the lack of an RS3 anchoring site, which weakens the attachment of the CPs. The radial spokes are considered to be related to the location/stability of the central bimodal. When DNAH1 is absent or dysfunctional, the axon is severely disorganized, usually as a $(9+0)$ structure (6). CP microtubule abnormalities are the most frequently observed ultrastructural abnormalities in genetically uncharacterized MMAF patients (26). Additionally, Abnormal sperm head morphology was present in $21.8 \%$ of MMAF patients with DNAH1 mutations (21). In DNAH1 knockout mice, TEM revealed a missing globule of the IDA3 head, resulting in a 3-2-1 arrangement (6). DNAHc1 (the ortholog of DNAH1) knockout mice showed decreased sperm motility and infertility (27). When most of sperm are alive but 
A

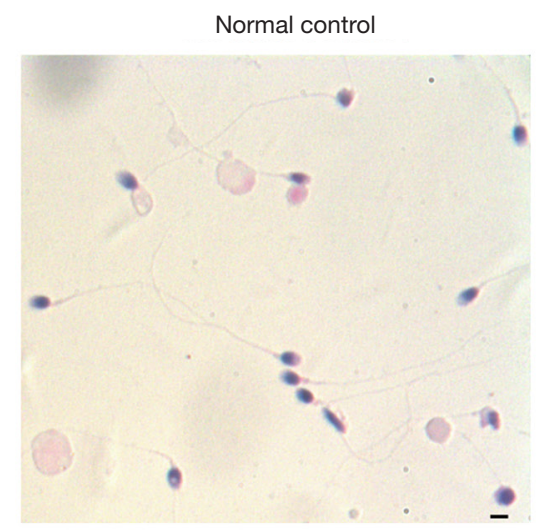

B

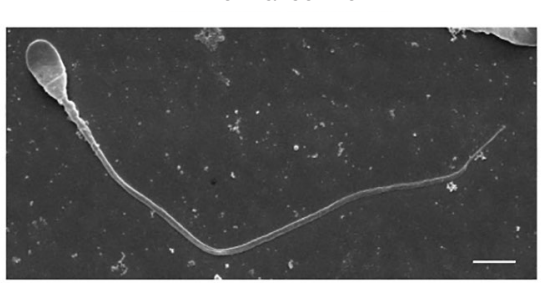

C
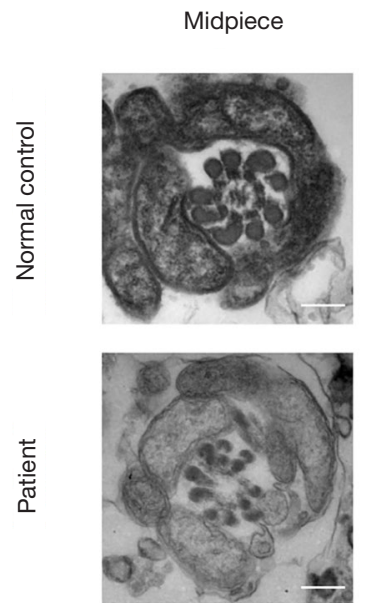

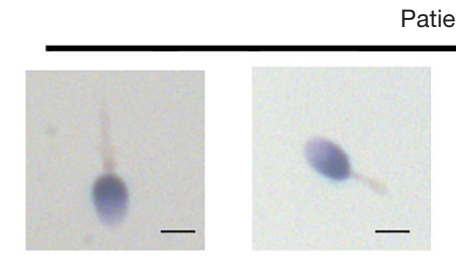

Patient
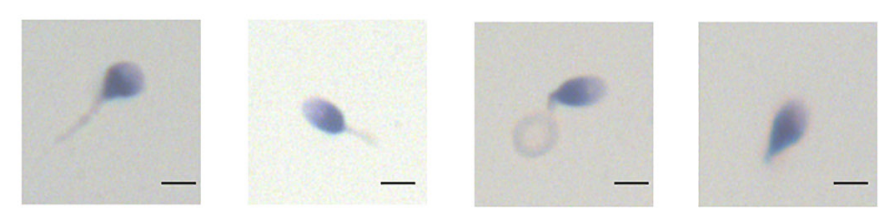

Patient
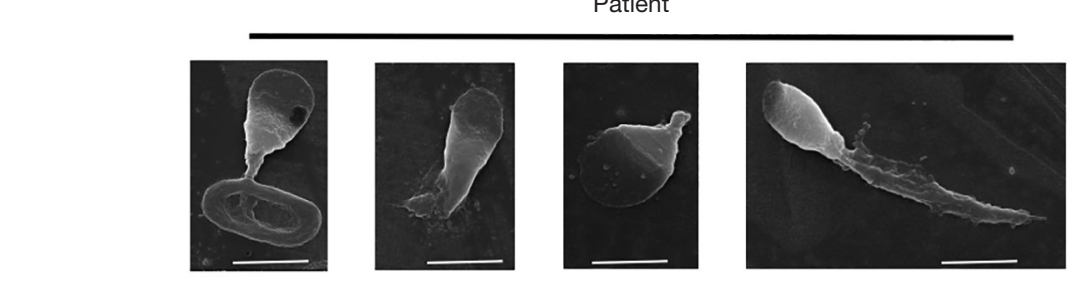

Prinpical piece

End piece
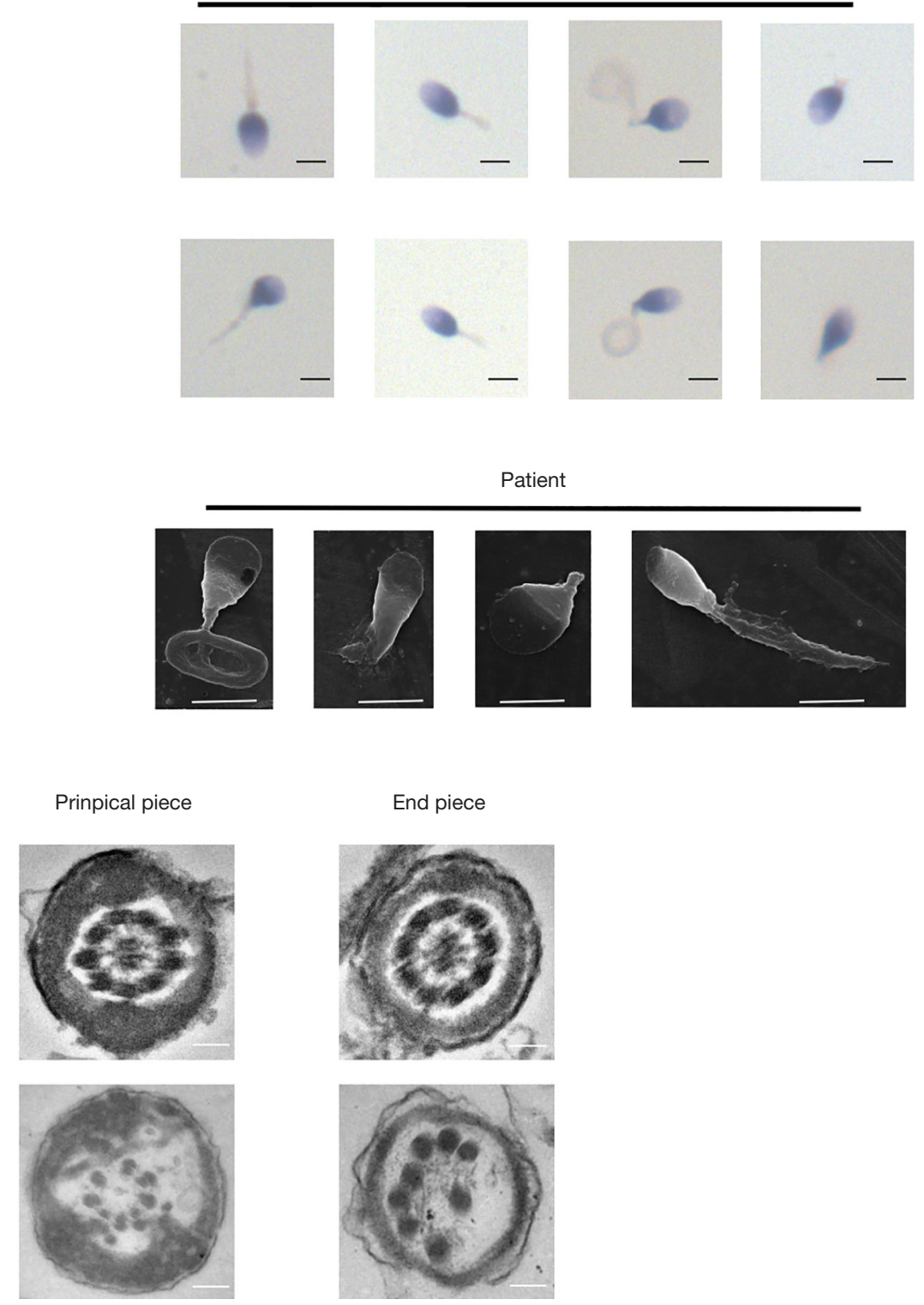

\section{.}

Figure 1 The MMAF phenotype was identified in the patient. (A) Papanicolaou staining of patient sperm cells showed short, absent, coiled flagella and irregular caliber (scale bars, $5 \mu \mathrm{m}$ ). (B) The patient sperm flagella showed typical MMAF phenotype by SEM (scale bars, $5 \mu \mathrm{m})$. (C) TEM results of the patient showed the abnormal ultrastructure of sperm flagellum: the central microtubule was missing, and the outer microtubule doublet was disordered arrangement in midpiece, principal piece, and end piece (scale bars, $100 \mathrm{~nm}$ ). MMAF, multiple morphological abnormalities of the flagella; SEM, scanning electron microscopy; TEM, transmission electron microscopy.

immotile, this is usually due to structural defects in the sperm flagellum (26). Variants introns 31 and 73 , and in exons 23 and 78 of the DNAH1 gene resulted in severe asthenospermia in MMAF without any PCD symptoms (6).

Mutated DNAH1 gene leads to various morphological abnormalities of sperm flagellum, causing male infertility
$(6,20,28,29)$. Mutant $D N A H 1$ variants are the first gene abnormalities to be universally acknowledged as causing MMAF and are estimated to account for up to a third of MMAF cases (6). The combination of DNAH1 gene mutations is supported by pedigree analysis: $52430998 \mathrm{CCT}>\mathrm{C}$ in exon 73, 52409336C $>\mathrm{T}$ in exon 
A 1

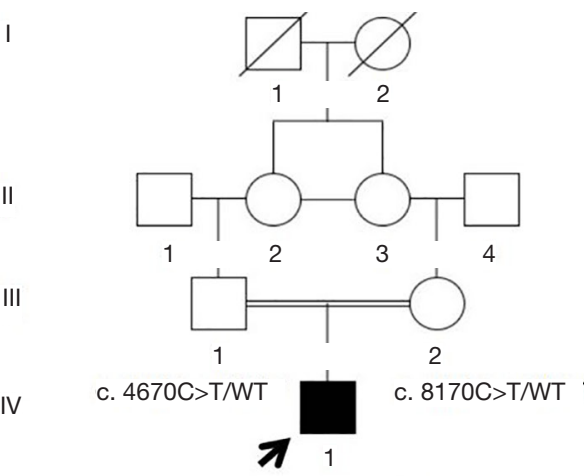

c. $4670 \mathrm{C}>\mathrm{T} / \mathrm{c} .8170 \mathrm{C}>\mathrm{T}$
B

Probandl

Father

Mather
NM_015512.4: c. 4670C>T, p. T1557M NM_015512.4: c. 8170.C>T, p. R2724X

CTGGTGATCACGCCCCTCACCO

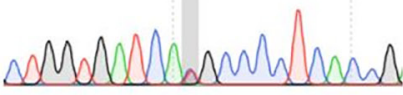

CTOGTOATCACOCCCCTCACCO

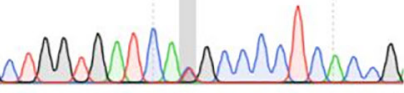

CTGOTGATCACGCCCCTCACCG

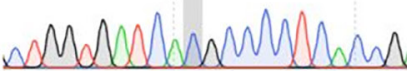
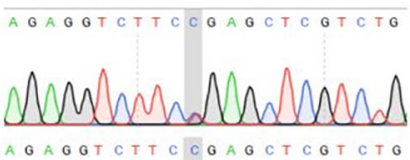

A GAGGTCTTCTGAGCTCGTCTG

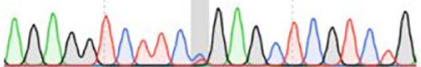

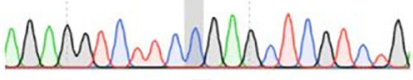

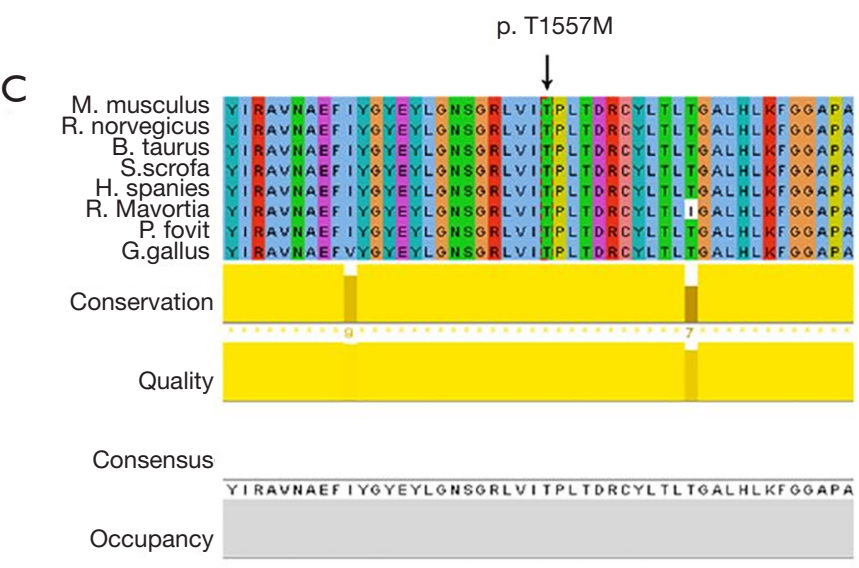

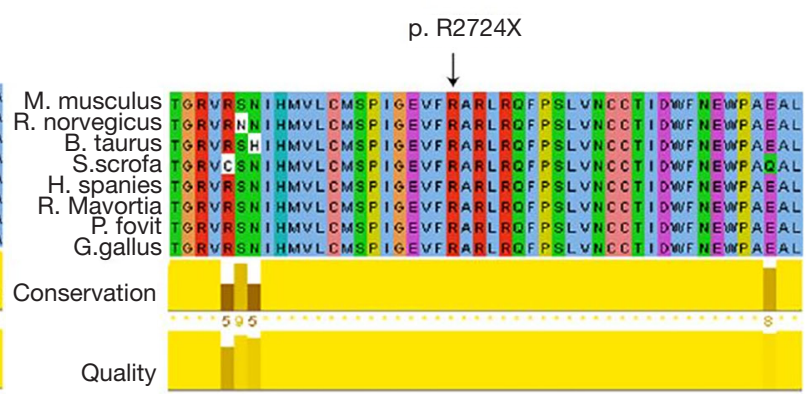

Consensus Occupancy IGRVRSN I HMVL CMSP I GEVF RARLRQF PSLUNCCI I DWF NEWPAEAL

Figure 2 The bi-allelic pathogenic mutations of DNAH1 in a consanguineous family. (A) Family pedigree. Squares represent male pedigree members, circles represent female pedigree members, and the black square represents the proband. Open symbols represent unaffected members. (B) Sanger sequencing of (c. 4607C>T) and (c. 8170C>T) were confirmed by PCR-sequencing in this family. (C) Multiple sequence alignment of the DNAH1 protein for different species. The black arrow denotes the position of the variants. PCR, polymerase chain reaction.

45 , and $52428484 \mathrm{G}>\mathrm{T}$ in exon 67 alone were related with MMAF and infertility in the ethnic Han Chinese population (22). A study reported that the homozygous frame-shift deletion DNAH1: exon73:c.11726_11727delCT caused MMAF, impairing sperm motility (23). This frameshift mutation resulted in the disorder of downstream amino acid sequence of 3909 aa and truncation of the DNAH1 protein. This severely damaged the dynein heavy chain domain at the c-terminal of the protein and the tertiary structure of DNAH1, resulting in its dysfunction (23). Three asthenoteratozoospermia patients, $2.09 \%$ of the patients tested using bioinformatics analyses, carried four pathogenic DNAH1 variants (5). The other two mutations were missense mutations, which were c. $6446 \mathrm{~T}>\mathrm{G}($ g. $52404762 \mathrm{~T}>\mathrm{G})$ and c. $7205 \mathrm{C}>\mathrm{A}(\mathrm{g}$. $52412624 \mathrm{C}>\mathrm{A}$ ), may adversely affect protein function (5).
Mouse with dynein heavy chain 7 (MDHC7) defects revealed that disruption of the $M D H C 7$ gene caused asthenozoospermia and reduced the frequency of cilia beats, but was not associated with any serious defects in axonemal structure. The data we present here in accordance with the previous studies of human HDHC7 $(6,28)$.

The progressive motility of sperm with DNAH1 mutations is higher than with other genetic causes for MMAF, ranging from $0 \%$ to $13 \%$ (25). Three independent semen tests performed within 6 months did not find any progressively motile sperm in our patient's samples; however, he had normal sperm survival rates. DNAH1 is highly expressed in testis and is necessary for the formation of the inner dynein arms (6). The absence of DNAH1 is very harmful to the organization and biogenesis of the axoneme. This result was expected given the important 
A
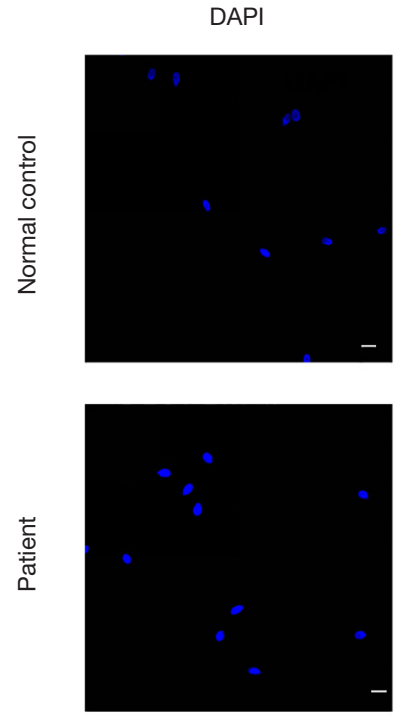

B

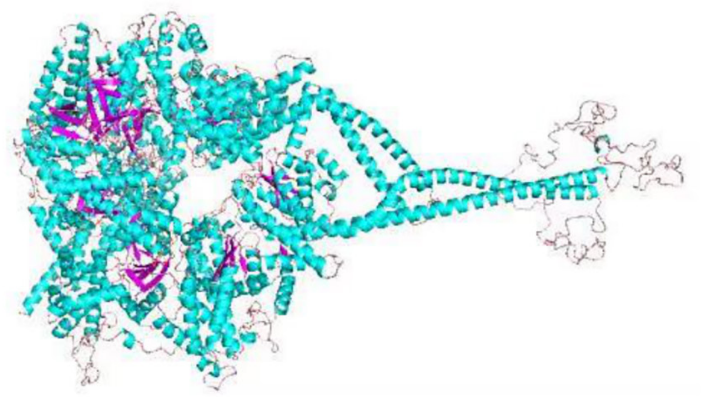

WT-DNAH1 $\alpha$-tubulin
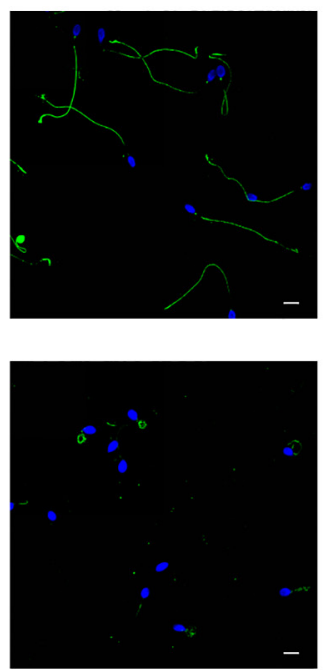

DNAH1
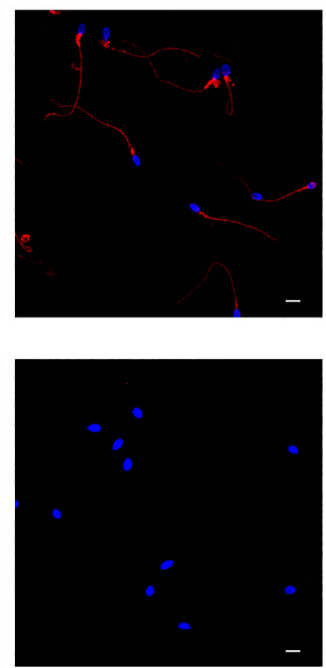

MERGE
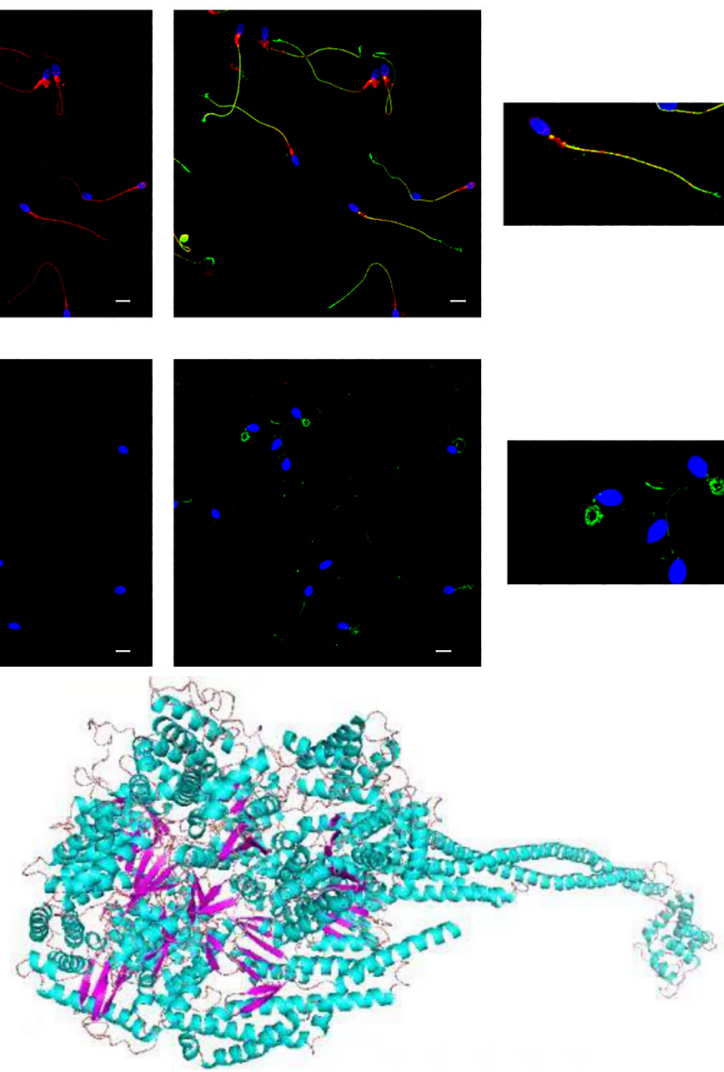

$\mathrm{DNAH1} 1^{\mathrm{T} 1557 \mathrm{M}}$
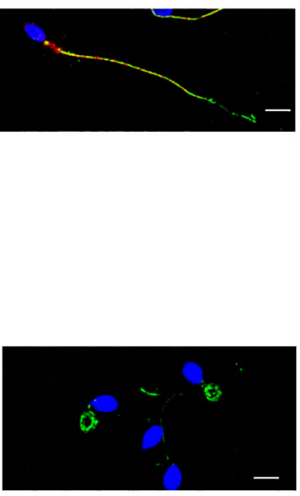

Figure 3 The influence of the bi-allelic mutations on DNAH1 protein. (A) Immunofluorescence staining in the sperm cells reveals an absence of DNAH1 protein in the patient compared to the normal control. (green, $\alpha$-tubulin; red, DNAH1; blue, DAPI; scale bars, $5 \mu$ m). (B) The spatial structure of DNAH1 was totally changed by the c. 4670C>T (p. T1557M) mutation, including random coils, $\alpha$-helix and $\beta$-sheet.

role of inner dynein arms in sperm motility. Although other dynein heavy chains may compensate for the absence of functional cilia owing to DNAH1 mutations in other structures, DNAH1 is critical for the proper functioning sperm flagella (6). Sperm flagellates and cilia, although similar in structure, differ significantly in axonal tissue and biogenesis (6). The role of DNAH1 in cilia may not be as crucial as it is in sperm flagella, and this may be evidenced by its lower levels of normal expression in other structures, including the trachea, compared to the testis. This allows the differentiation between DNAH1 mutant MMAF patients and PCD patients because PCD typically has other severe symptoms in addition to infertility. Collectively, this supports our findings that DNAH1 is essential for flagellar development in sperm.

In conclusion, in this study, we identified two novel bi- allelic pathogenic mutations of $D N A H 1$ gene in an infertile male, thus expanding the known spectrum of $D N A H 1$ gene mutations, further helping identify the pathogenesis and causative variation affecting MMAF-induced male infertility, finally providing beneficial diagnosis and prognosis for male infertility.

\section{Acknowledgments}

The authors would like to thank the Analytical and Testing center of Sichuan University for Guiping Yuan for her help of TEM images. The authors would like to thank Mr. Romel Wizar for language editing.

Funding: This work was supported by the Xinya Foundation of West China Second University Hospital (KX094 \& KX249). 


\section{Footnote}

Reporting Checklist: The authors have completed the MDAR reporting checklist. Available at http://dx.doi.org/10.21037/ tau-20-1434

Data Sharing Statement: Available at http://dx.doi. org/10.21037/tau-20-1434

Conflicts of Interest: All authors have completed the ICMJE uniform disclosure form (available at http://dx.doi. org/10.21037/tau-20-1434). The authors have no conflicts of interest to declare.

Ethical Statement: The authors are accountable for all aspects of the work in ensuring that questions related to the accuracy or integrity of any part of the work are appropriately investigated and resolved. The study was conducted in accordance with the Declaration of Helsinki (as revised in 2013). The study was approved by the ethics committee of West China Second University Hospital (No. 2019019) and informed consent was taken from the patient and all members of his family.

Open Access Statement: This is an Open Access article distributed in accordance with the Creative Commons Attribution-NonCommercial-NoDerivs 4.0 International License (CC BY-NC-ND 4.0), which permits the noncommercial replication and distribution of the article with the strict proviso that no changes or edits are made and the original work is properly cited (including links to both the formal publication through the relevant DOI and the license). See: https://creativecommons.org/licenses/by-nc-nd/4.0/.

\section{References}

1. Ferlin A. New genetic markers for male fertility. Asian J Androl 2012;14:807-8.

2. Silflow CD, Lefebvre PA. Assembly and motility of eukaryotic cilia and flagella. Lessons from Chlamydomonas reinhardtii. Plant Physiol 2001;127:1500-7.

3. Zhang X, Shen Y, Wang X, et al. A novel homozygous CFAP65 mutation in humans causes male infertility with multiple morphological abnormalities of the sperm flagella. Clin Genet 2019;96:541-8.

4. Shen Y, Zhang F, Li F, et al. Loss-of-function mutations in QRICH2 cause male infertility with multiple morphological abnormalities of the sperm flagella.
Nat Commun 2019;10:433. Erratum in: Nat Commun 2019;10:2289.

5. Yang $X$, Zhu D, Zhang H, et al. Associations between DNAH1 gene polymorphisms and male infertility: a retrospective study. Medicine (Baltimore) 2018;97:e13493.

6. Ben Khelifa M, Coutton C, Zouari R, et al. Mutations in DNAH1, which encodes an inner arm heavy chain dynein, lead to male infertility from multiple morphological abnormalities of the sperm flagella. Am J Hum Genet 2014;94:95-104.

7. Sha YW, Wang X, Su ZY, et al. Patients with multiple morphological abnormalities of the sperm flagella harbouring CFAP44 or CFAP43 mutations have a good pregnancy outcome following intracytoplasmic sperm injection. Andrologia 2019;51:e13151.

8. Tang $\mathrm{S}$, Wang $\mathrm{X}, \mathrm{Li} \mathrm{W}$, et al. Biallelic mutations in CFAP43 and CFAP44 cause male infertility with multiple morphological abnormalities of the sperm flagella. Am J Hum Genet 2017;100:854-64.

9. $\mathrm{He} \mathrm{X}, \mathrm{Li} \mathrm{W}, \mathrm{Wu} \mathrm{H}$, et al. Novel homozygous CFAP69 mutations in humans and mice cause severe asthenoteratospermia with multiple morphological abnormalities of the sperm flagella. J Med Genet 2019;56:96-103.

10. Kherraf ZE, Amiri-Yekta A, Dacheux D, et al. A homozygous ancestral SVA-insertion-mediated deletion in WDR66 induces multiple morphological abnormalities of the sperm flagellum and male infertility. Am J Hum Genet 2018;103:400-12.

11. Auguste Y, Delague V, Desvignes JP, et al. Loss of calmodulin- and radial-spoke-associated complex protein CFAP251 leads to immotile spermatozoa lacking mitochondria and infertility in men. Am J Hum Genet 2018;103:413-20.

12. Lorès $\mathrm{P}$, Coutton $\mathrm{C}, \mathrm{El} \mathrm{KE}$, et al. Homozygous missense mutation L673P in adenylate kinase 7 (AK7) leads to primary male infertility and multiple morphological anomalies of the flagella but not to primary ciliary dyskinesia. Hum Mol Genet 2018;27:1196-211.

13. Sha YW, Xu X, Mei LB, et al. A homozygous CEP135 mutation is associated with multiple morphological abnormalities of the sperm flagella (MMAF). Gene 2017;633:48-53.

14. Martinez G, Kherraf ZE, Zouari R, et al. Whole-exome sequencing identifies mutations in FSIP2 as a recurrent cause of multiple morphological abnormalities of the sperm flagella. Hum Reprod 2018;33:1973-84.

15. Liu W, Sha Y, Li Y, et al. Loss-of-function mutations in 
SPEF2 cause multiple morphological abnormalities of the sperm flagella (MMAF). J Med Genet 2019;56:678-84.

16. Liu $W, \mathrm{He} X$, Yang $\mathrm{S}$, et al. Bi-allelic mutations in TTC21A induce asthenoteratospermia in humans and mice. Am J Hum Genet 2019;104:738-48.

17. Coutton C, Martinez G, Kherraf ZE, et al. Biallelic mutations in ARMC2 lead to severe asthenoteratozoospermia due to sperm flagellum malformations in humans and mice. Am J Hum Genet 2019;104:331-40.

18. Merveille AC, Davis EE, Becker-Heck A, et al. CCDC39 is required for assembly of inner dynein arms and the dynein regulatory complex and for normal ciliary motility in humans and dogs. Nat Genet 2011;43:72-8.

19. Liu C, Lv M, He X, et al. Homozygous mutations in SPEF2 induce multiple morphological abnormalities of the sperm flagella and male infertility. J Med Genet 2020;57:31-7.

20. Wambergue C, Zouari R, Fourati BMS, et al. Patients with multiple morphological abnormalities of the sperm flagella due to DNAH1 mutations have a good prognosis following intracytoplasmic sperm injection. Hum Reprod 2016;31:1164-72.

21. Amiri-Yekta A, Coutton C, Kherraf ZE, et al. Wholeexome sequencing of familial cases of multiple morphological abnormalities of the sperm flagella (MMAF) reveals new DNAH1 mutations. Hum Reprod 2016;31:2872-80.

22. Sha Y, Yang X, Mei L, et al. DNAH1 gene mutations

Cite this article as: Jiang C, Zhang X, Zhang H, Guo J, Zhang C, Li J, Yang Y. Novel bi-allelic mutations in DNAH1 cause multiple morphological abnormalities of the sperm flagella resulting in male infertility. Transl Androl Urol 2021;10(4):1656-1664. doi: 10.21037/tau-20-1434 and their potential association with dysplasia of the sperm fibrous sheath and infertility in the Han Chinese population. Fertil Steril 2017;107:1312-8.e2.

23. Wang X, Jin H, Han F, et al. Homozygous DNAH1 frameshift mutation causes multiple morphological anomalies of the sperm flagella in Chinese. Clin Genet 2017;91:313-21.

24. Coutton C, Arnoult C, Ray PF. Commentary on "morphological characteristics and initial genetic study of multiple morphological anomalies of the flagella in China". Asian J Androl 2016;18:812.

25. Wang WL, Tu CF, Tan YQ. Insight on multiple morphological abnormalities of sperm flagella in male infertility: what is new. Asian J Androl 2020;22:236-45.

26. Chemes EH, Rawe YV. Sperm pathology: a step beyond descriptive morphology. Origin, characterization and fertility potential of abnormal sperm phenotypes in infertile men. Hum Reprod Update 2003;9:405-28.

27. Neesen J, Kirschner R, Ochs M, et al. Disruption of an inner arm dynein heavy chain gene results in asthenozoospermia and reduced ciliary beat frequency. Hum Mol Genet 2001;10:1117-28.

28. Imtiaz F, Allam R, Ramzan K, et al. Variation in DNAH1 may contribute to primary ciliary dyskinesia. BMC Med Genet 2015;16:14.

29. Coutton C, Escoffier J, Martinez G, et al. Teratozoospermia: spotlight on the main genetic actors in the human. Hum Reprod Update 2015;21:455-85. 